\title{
Duração das alterações em propriedades físico-hídricas de Latossolo argiloso decorrentes da escarificação mecânica
}

\author{
Marta Sandra Drescher(1), Dalvan José Reinert(2), José Eloir Denardin ${ }^{(3)}$, Paulo Ivonir Gubiani(2), \\ Antônio Faganello ${ }^{(3)}$ e Gerson Laerson Drescher(2)
}

\begin{abstract}
(1)Universidade Estadual do Rio Grande do Sul, Rua Marechal Floriano, no 4.557, Bairro Agrícola, CEP 97800-000 São Luiz Gonzaga, RS, Brasil. E-mail: marta-drescher@uergs.edu.br (2)Universidade Federal de Santa Maria, Centro de Ciências Rurais, Departamento de Solos, Avenida Roraima, № 1.000, Cidade Universitária, Bairro Camobi, CEP 97105-900 Santa Maria, RS, Brasil. E-mail: dalvan@ufsm.br, paulogubiani@gmail.com, gersondrescher@gmail.com ${ }^{(3)}$ Embrapa Trigo, Rodovia BR-285, Km 294, Caixa Postal 3081, CEP 99050-970 Passo Fundo, RS, Brasil. E-mail: jose.denardin@embrapa.br, antonio.faganello@embrapa.br
\end{abstract}

Resumo - O objetivo deste trabalho foi determinar o tempo de duração do efeito da descompactação do solo, por escarificação mecânica, por meio de indicadores físico-hídricos de Latossolo argiloso, manejado em sistema plantio direto (SPD). Os tratamentos consistiram em meses $(0,6,12,18,24,30$ e 36) transcorridos após escarificação mecânica em SPD e em testemunha sem escarificação em SPD há 27 anos. Foram avaliadas as variáveis: resistência mecânica à penetração, taxa de infiltração de água no solo, densidade e densidade relativa do solo, distribuição do tamanho de poros e condutividade hidráulica do solo saturado. A duração dos efeitos da escarificação mecânica variou com a propriedade do solo, tendo se mantido por 6 meses na densidade e na densidade relativa, na porosidade total e na macroporosidade; 18 meses na resistência à penetração; e 24 meses na condutividade hidráulica e na taxa de infiltração de água no solo. Propriedades do solo relacionadas ao transporte de água, como condutividade hidráulica e taxa de infiltração estável de água no solo, mantêm o efeito da escarificação por mais tempo e, portanto, são mais adequadas para avaliar a duração da descompactação mecânica.

Termos para indexação: compactação, descompactação mecânica, estrutura do solo, manejo do solo.

\section{Duration of changes in physical and hydraulic properties of a clayey Oxisol by mechanical chiseling}

\begin{abstract}
The objective of this work was to determine the duration of the effects of soil decompaction, by mechanical chiseling, through physical and hydraulic indicators of a clayey Oxisol under no-tillage (NT). The treatments consisted of months $(0,6,12,18,24,30$, and 36) after chiseling under NT and of a control treatment without chiseling under NT during 27 years. The following variables were evaluated: penetration resistance, infiltration rate, bulk density and relative density, pore size distribution, and saturated hydraulic conductivity. The duration of the effects of mechanical chiseling varied according to the evaluated soil property, lasting six months for bulk density and relative density, total porosity, and macroporosity; 18 months for penetration resistance; and 24 months for hydraulic conductivity and infiltration rate. Soil properties related to water transport, such as hydraulic conductivity and steady infiltration rate, maintain the effect of mechanical chiseling for a longer time and, therefore, are more suitable to measure the duration of mechanical decompaction.
\end{abstract}

Index terms: soil compaction, mechanical decompaction, soil structure, soil management.

\section{Introdução}

O entendimento do processo de compactação do solo e a busca por alternativas para mitigar seus efeitos têm motivado a realização de estudos em escala global, uma vez que a compactação é considerada uma das principais ameaças à manutenção da qualidade do solo em áreas agrícolas (Schjønning et al., 2009; Keller et al., 2013). No cenário agrícola brasileiro, áreas manejadas em plantio direto ocupam mais de 30 milhões do total de 48,8 milhões de hectares cultivados com espécies temporárias (Federação Brasileira de Plantio Direto na Palha, 2014). Nessas áreas, há registros de aumento da compactação do solo na camada localizada entre 0,07 e 0,15 m (Suzuki et al., 2008; Drescher et al., 2011), decorrente de descuidos no manejo, como ausência de diversificação de culturas, excesso de calagem, uso de semeadoras desprovidas de hastes sulcadoras 
com deposição de adubo na superfície ou na camada superficial do solo, pastejo intensivo e aumento da frequência de tráfego de máquinas agrícolas (Denardin et al., 2011).

NaregiãoSul doPaís, mais de $80 \%$ daárea de produção de grãos é cultivada com soja (Glycine max L.), milho (Zea mays L.) e trigo (Triticum aestivum L.) em plantio direto (Cooperativa dos Agricultores de Plantio Direto, 2012). Nessas áreas, o aumento da compactação do solo tem provocado restrição ao crescimento radicular das plantas e consequente concentração de raízes na camada superficial do solo, o que pode resultar na diminuição da absorção de água e nutrientes, mesmo em curtos períodos de deficit hídrico, e na redução do crescimento da parte aérea e da produtividade das culturas (Klein et al., 2009).

Como estratégia para descompactar ou retardar o aumento da compactação do solo, habitualmente utilizam-se máquinas para a escarificação (Jin et al., 2007). Entretanto, o uso dessa prática deve ser limitado, pois requer elevado consumo energético, investimento em equipamentos e mudanças no sistema de cultivo (Chamen, 2015), em que os custos aumentam proporcionalmente à profundidade da camada compactada, e os benefícios dependem da textura do solo e da profundidade e da frequência da operação. Além disso, os benefícios da escarificação do solo sobre o rendimento de culturas nem sempre se concretizam (Gubiani et al., 2013; Nunes et al., 2014).

Outra limitação do uso da escarificação como estratégia de recuperação da estrutura do solo é a efemeridade dos benefícios sobre as propriedades do solo, os quais, muitas vezes, duram menos de 1 ano (Álvarez et al., 2009). Contudo, diversos autores relataram que a duração do efeito da escarificação pode se estender por mais de 2 anos (Botta et al., 2006; Drescher et al., 2011), de acordo com as características e as propriedades do solo, e com as práticas de manejo a serem utilizadas subsequentemente à escarificação.

Além das características do solo e das práticas de manejo, as divergências na duração dos benefícios da escarificação também se relacionam às propriedades do solo. Em trabalhos com variáveis indicadoras do estado estrutural do solo, como densidade e porosidade, observou-se duração inferior a 1 ano (Drescher et al., 2012; Silva et al., 2012), mas, em avaliações das propriedades físico-hídricas, diretamente relacionadas a processos do solo, como condutividade hidráulica, verificou-se a manutenção dos efeitos da escarificação por aproximadamente 24 meses (Vieira \& Klein, 2007).

Assim, a escolha da propriedade do solo que será avaliada é ponto-chave para determinar a duração dos efeitos da escarificação e permitir a compreensão de processos diretamente associados à variável escolhida. Desse modo, os resultados obtidos podem ampliar ou diminuir o período no qual se afirma existir efeito da escarificação.

O objetivo do presente trabalho foi determinar o tempo de duração do efeito da descompactação do solo, por escarificação mecânica, por meio de indicadores físico-hídricos de Latossolo argiloso, manejado em sistema plantio direto.

\section{Material e Métodos}

O estudo foi realizado no campo experimental da Embrapa Trigo, localizado no município de Coxilha, RS. O clima, de acordo com a classificação de Köppen, é do tipo $\mathrm{Cfa}$, subtropical úmido, e o solo é classificado como Latossolo Vermelho distrófico típico (Santos et al., 2013), de textura argilosa (Tabela 1), profundo e bem drenado, localizado em relevo ondulado a suavemente ondulado.

O experimento foi realizado de 2009 a 2012, em área de produção de grãos manejada em sistema plantio direto (SPD) há 27 anos, ou seja, desde 1986. Considerou-se como tratamento o tempo transcorrido após a escarificação mecânica em SPD $\left(\mathrm{E}_{\text {tempo }}\right)$ : E0, E6, E12, E18, E24, E30 e E36, em que os números representam os meses transcorridos após a escarificação, que foram comparados ao tratamento testemunha, constituído pelo SPD contínuo há 27 anos $\left(\mathrm{SPD}_{\text {cont }}\right)$. As escarificações foram realizadas até $0,25 \mathrm{~m}$ de profundidade, com escarificador equipado com cinco hastes, espaçadas em $0,30 \mathrm{~m}$, e rolo destorroador, que dispensa a operação de gradagem subsequente. $\mathrm{O}$ experimento foi conduzido em delineamento de blocos ao acaso, com quatro repetições, o que totalizou 32 unidades experimentais de $14,4 \mathrm{~m}^{2}(8,0 \times 1,8 \mathrm{~m})$.

No período compreendido entre as safras agrícolas de verão 2009/2010 e 2012/2013, a área experimental foi cultivada com a sequência de culturas: milho/ trigo, soja/centeio (Secale cereale L.), milho/trigo e soja. As culturas foram implantadas com semeadora equipada com discos duplos defasados, para atuar a 0,07 $\mathrm{m}$ de profundidade. 
Em dezembro de 2012 e abril de 2013 (início e fim, respectivamente, do ciclo da cultura de soja, safra 2012/2013), a resistência mecânica do solo à penetração (RP) foi medida em campo, com uso de penetrômetro georreferenciado PNT-2000 (DLG Automação Industrial, Sertãozinho, SP), de acordo com a norma ASAE S313.3. A haste com ponta cônica de $30^{\circ}$ e com área do cone de $129 \mathrm{~mm}^{2}$ foi inserida no solo com velocidade de penetração de, aproximadamente, 2,0 $\mathrm{m} \mathrm{min}^{-1}$, e os dados de RP foram registrados a cada $0,01 \mathrm{~m}$, até $0,40 \mathrm{~m}$ de profundidade. As medições da RP foram realizadas na linha de semeadura e a 0,05 , 0,10 e $0,20 \mathrm{~m}$ à esquerda e à direita, na entrelinha de semeadura. No momento das medições da RP, foram coletadas amostras de solo, para determinação do conteúdo gravimétrico de água, a 0,03, 0,10, 0,15, $0,20,0,30$ e $0,40 \mathrm{~m}$ de profundidade, em três pontos de cada bloco experimental.

A taxa de infiltração de água no solo foi medida em março de 2013, com infiltrômetro de anéis concêntricos, com diâmetros de 0,20 e 0,40 m para anel interno e externo, respectivamente, ambos inseridos no solo até a profundidade de $0,15 \mathrm{~m}$, seguindo método descrito em Claessen (1997). Considerou-se como taxa de infiltração estável (TIE) a da última leitura, às 2 horas, em razão da pequena variação da taxa de infiltração, nesse tempo.

Amostras de solo com estrutura preservada foram coletadas com cilindros de aço inox de $0,04 \times 0,055 \mathrm{~m}$, em maio de 2013, para determinação da densidade e da porosidade do solo. As amostras foram coletadas na entrelinha de semeadura da soja, safra 2012/2013, em três profundidades, tendo-se utilizado, para a definição das camadas, a avaliação do perfil cultural, como em Tavares Filho et al. (1999). De acordo com este método, consideraram-se, como camada superficial, a que tinha estrutura granular solta e apresentava maior concentração de raízes, aproximadamente de 0 a $0,07 \mathrm{~m}$; como segunda camada, aquela que apresentava estrutura maciça, na qual se percebia reduzida concentração de raízes, aproximadamente de 0,07 a $0,15 \mathrm{~m}$; e, como terceira camada, aquela que apresentava estrutura típica de Latossolo, com pequena presença de raízes, aproximadamente de 0,15 a $0,25 \mathrm{~m}$.

Após a coleta, as amostras foram saturadas por capilaridade por 48 horas, pesadas e levadas à coluna de areia (Reinert \& Reichert, 2006); em seguida, foram submetidas à tensão de $6,0 \mathrm{kPa}$ por 48 horas, pesadas e, posteriormente, ressaturadas e submetidas à análise de condutividade hidráulica, a qual foi avaliada em permeâmetro de carga constante, de acordo com Libardi (2012). A condutividade hidráulica (Ks) foi calculada pelo rearranjo da equação de Darcy: $\mathrm{Ks}=\mathrm{VH} / \operatorname{At}(\mathrm{H}+\mathrm{h})$, em que: V $\left(\mathrm{cm}^{3}\right)$ é o volume de água que se desloca ao longo da amostra $\mathrm{H}(\mathrm{cm})$ e passa pela área da seção transversal $\mathrm{A}\left(\mathrm{cm}^{2}\right)$ da amostra, no intervalo de tempo $\mathrm{t}$ (horas); e h é a altura da lâmina de água sobre a amostra $(\mathrm{cm})$.

A porosidade total $(\mathrm{Pt})$, a microporosidade, a macroporosidade e a densidade do solo (Ds) foram determinadas pelo método adotado por Claessen (1997). A densidade relativa (Dr) foi calculada com a divisão da Ds pela densidade máxima do solo ( $\left.\mathrm{Ds}_{\text {máx }}\right)$. $\mathrm{A} \mathrm{Ds}_{\text {máx }}$ foi estimada por meio da equação de Marcolin \& Klein (2011): $\mathrm{Ds}_{\text {máx }}=-0,0092$ Argila $+2,0138$ $\left(r^{2}=0,92\right)$, com argila em percentagem, tendo-se obtido valores de $1,52,1,51 \mathrm{e} 1,50 \mathrm{Mg} \mathrm{m}^{-3}$, respectivamente, para as camadas de $0-0,07,0,07-0,15$ e $0,15-0,25 \mathrm{~m}$.

As variáveis foram submetidas aos testes de Lilliefors para normalidade e de Cochran para homogeneidade de variâncias. Detectou-se distribuição não normal da Ks e da TIE, em que a transformação logarítmica foi capaz de normalizar apenas a TIE. A distribuição da Ks continuou não normal mesmo após a aplicação de outras transformações. Por isso, a Ks foi analisada pelo teste não paramétrico de Kruskal-Wallis, e os postos dos valores das variáveis, após ordenação, foram comparados pelo teste t, a $5 \%$ de probabilidade (McDonald, 2014). As demais variáveis foram submetidas à análise de variância, a 5\% de

Tabela 1. Composição granulométrica e densidade de partículas de Latossolo Vermelho distrófico típico da área experimental.

\begin{tabular}{lccccc}
\hline $\begin{array}{l}\text { Camada } \\
(\mathrm{m})\end{array}$ & Areia grossa & Areia fina & Silte & $\begin{array}{c}\text { Argila } \\
\left(\mathrm{g} \mathrm{kg}^{-1}\right)\end{array}$ & $\begin{array}{c}\text { Densidade de partículas } \\
\left(\mathrm{Mg} \mathrm{m}^{-3}\right)\end{array}$ \\
\hline $0,0-0,07$ & 66 & 163 & 231 & 540 & 2,79 \\
$0,07-0,15$ & 59 & 162 & 230 & 549 & 2,79 \\
$0,15-0,25$ & 57 & 142 & 246 & 555 & 2,77 \\
\hline
\end{tabular}


probabilidade. Quando o teste $\mathrm{F}$ foi significativo, os resultados foram comparados pelo teste de Dunnett, também a $5 \%$ de probabilidade. Todas as avaliações foram realizadas no programa estatístico Assistat, versão 7.7 beta (Universidade Federal de Campina Grande, Campina Grande, PB).

\section{Resultados e Discussão}

Alterações na Ds, na Dr, na Pt e na macroporosidade decorrentes da escarificação em SPD foram mantidas apenas na primeira safra agrícola - E0 (Tabela 2), na camada de 0,07 a $0,15 \mathrm{~m}$; nas demais camadas avaliadas, não houve diferença entre os períodos de tempo após escarificação. Silva et al. (2012) também observaram efeitos da escarificação na Ds com tempo de duração inferior a 1 ano, em Latossolo Vermelho distrófico. A rápida reconsolidação da estrutura do solo, como indicam as propriedades Ds, Pt, macroporosidade e microporosidade, pode acarretar necessidade de repetição semestral de descompactação mecânica, o que se contrapõe aos fundamentos do SPD, uma vez que o uso frequente da escarificação pode romper agregados e acelerar a mineralização da matéria orgânica do solo, o que torna o solo mais suscetível à compactação subsequente em maior intensidade (Canarache et al., 2000).

É importante ressaltar que a duração dos efeitos da escarificação pode ser intensificada ou retardada, dependentemente da intensidade da manifestação de fatores controladores da reconsolidação e da resiliência do solo, como precipitações, ciclos de umedecimento e secagem, e espécies cultivadas, particularmente quando ocorrem imediatamente após uma determinada escarificação. Contudo, esses fatores não podem ser controlados nesse tipo de experimento executado em campo.

A RP avaliada logo após a semeadura da soja (Figura 1) foi menor do que a avaliada no final do ciclo da cultura (Figura 2), embora o conteúdo de água nas camadas superficiais do solo tenha sido maior no final do ciclo (Figura 3). No presente trabalho, não houve variação do conteúdo de água no solo entre os tratamentos, como evidenciado pelos baixos valores de desvio-padrão obtidos para essa variável; portanto, discutiu-se apenas o efeito da densidade. Isso porque, no momento das medições de RP, no início e no fim do ciclo da soja, safra 2012/2013, houve pequena variação da umidade entre as unidades experimentais e as camadas avaliadas, em decorrência de chuvas ocorridas 2 dias antes do momento das medições.

Em todos os tratamentos, a RP dos primeiros $0,05 \mathrm{~m}$ foi inferior a 1,0 MPa, o que pode ter sido consequência da maior atuação dos ciclos de umedecimento e de secagem, bem como da atividade biológica, associada à recente ação dos discos da semeadora. Em profundidades superiores a $0,07 \mathrm{~m}$, onde não houve atuação dos discos da semeadora, verificouse incremento da RP, o que confirma a existência de uma camada mais compactada, localizada entre 0,07

Tabela 2. Densidade do solo (Ds), densidade relativa (Dr), porosidade total $(\mathrm{Pt})$, micro $(\mathrm{Mi})$ e macroporosidade $(\mathrm{Ma}) \mathrm{de}$ Latossolo Vermelho distrófico típico, manejado em sistema plantio direto contínuo há 27 anos $\left(\mathrm{SPD}_{\text {cont }}\right)$ e em SPD escarificado mecanicamente há 0 (E0), 6 (E6), 12 (E12), 18 (E18), 24 (E24), 30 (E30) e 36 (E36) meses $^{(1)}$.

\begin{tabular}{|c|c|c|c|c|c|}
\hline \multirow[t]{3}{*}{ Tratamento } & Ds & $\mathrm{Dr}$ & $\mathrm{Pt}$ & $\mathrm{Mi}$ & $\mathrm{Ma}$ \\
\hline & \multicolumn{2}{|c|}{-------($\left(\mathrm{Mg} \mathrm{m}^{-3}\right)$-------- } & \multicolumn{3}{|c|}{---------------- $\left(\mathrm{m}^{3} \mathrm{~m}^{-3}\right)$---------------. } \\
\hline & \multicolumn{5}{|c|}{ Camada 0,0 a $0,07 \mathrm{~m}$ de profundidade } \\
\hline E0 & $1,18^{\mathrm{ns}}$ & $0,78^{\mathrm{ns}}$ & $0,59^{\text {ns }}$ & $0,38^{\mathrm{ns}}$ & $0,21^{\mathrm{ns}}$ \\
\hline E6 & 1,20 & 0,79 & 0,57 & 0,37 & 0,21 \\
\hline E12 & 1,23 & 0,81 & 0,56 & 0,36 & 0,19 \\
\hline E18 & 1,22 & 0,80 & 0,57 & 0,40 & 0,16 \\
\hline E24 & 1,32 & 0,87 & 0,52 & 0,41 & 0,11 \\
\hline $\mathrm{E} 30$ & 1,32 & 0,87 & 0,54 & 0,43 & 0,11 \\
\hline E36 & 1,24 & 0,82 & 0,57 & 0,40 & 0,17 \\
\hline$\underline{\mathrm{SPD}_{\text {cont }}}$ & 1,24 & 0,82 & 0,54 & 0,40 & 0,14 \\
\hline \multirow[t]{2}{*}{$\mathrm{CV}(\%)$} & 7,8 & 7,8 & 9,5 & 9,7 & 43,8 \\
\hline & \multicolumn{5}{|c|}{ Camada de 0,07 a $0,15 \mathrm{~m}$ de profundidade } \\
\hline E0 & $1,30 \mathrm{a}$ & $0,86 \mathrm{a}$ & $0,55 \mathrm{a}$ & $0,40^{\mathrm{ns}}$ & $0,15 \mathrm{a}$ \\
\hline E6 & $1,39 b$ & $0,92 b$ & $0,51 b$ & 0,40 & $0,11 b$ \\
\hline E12 & $1,38 b$ & $0,91 b$ & $0,51 b$ & 0,40 & $0,12 \mathrm{~b}$ \\
\hline E18 & $1,41 b$ & $0,94 b$ & $0,50 \mathrm{~b}$ & 0,40 & $0,10 \mathrm{~b}$ \\
\hline E24 & $1,36 \mathrm{~b}$ & $0,90 \mathrm{~b}$ & $0,49 b$ & 0,39 & $0,11 \mathrm{~b}$ \\
\hline E30 & $1,39 b$ & $0,92 b$ & $0,50 \mathrm{~b}$ & 0,40 & $0,10 \mathrm{~b}$ \\
\hline E36 & $1,38 b$ & $0,92 b$ & $0,50 \mathrm{~b}$ & 0,40 & $0,10 \mathrm{~b}$ \\
\hline$\underline{\mathrm{SPD}_{\text {cont }}}$ & $1,43 \mathrm{~b}$ & $0,95 \mathrm{~b}$ & $0,48 \mathrm{~b}$ & 0,41 & $0,07 \mathrm{~b}$ \\
\hline \multirow[t]{2}{*}{ CV (\%) } & 3,7 & 3,71 & 3,8 & 3,8 & 23,6 \\
\hline & \multicolumn{5}{|c|}{ Camada de 0,15 a $0,25 \mathrm{~m}$ de profundidade } \\
\hline E0 & $1,33^{\mathrm{ns}}$ & $0,89^{\text {ns }}$ & $0,51^{\mathrm{ns}}$ & $0,45^{\mathrm{ns}}$ & $0,06 \mathrm{~b}$ \\
\hline E6 & 1,29 & 0,86 & 0,52 & 0,46 & $0,06 \mathrm{~b}$ \\
\hline E12 & 1,29 & 0,86 & 0,53 & 0,45 & $0,08 \mathrm{~b}$ \\
\hline E18 & 1,22 & 0,81 & 0,54 & 0,43 & $0,11 \mathrm{a}$ \\
\hline E24 & 1,34 & 0,89 & 0,50 & 0,44 & $0,06 \mathrm{~b}$ \\
\hline E30 & 1,30 & 0,86 & 0,51 & 0,45 & $0,07 \mathrm{~b}$ \\
\hline E36 & 1,26 & 0,84 & 0,54 & 0,45 & $0,09 \mathrm{~b}$ \\
\hline$\underline{\mathrm{SPD}_{\text {cont }}}$ & 1,34 & 0,89 & 0,51 & 0,44 & $0,07 \mathrm{~b}$ \\
\hline $\mathrm{CV}(\%)$ & 5,3 & 5,3 & 4,0 & 3,5 & 24,2 \\
\hline
\end{tabular}


e $0,15 \mathrm{~m}$ em áreas em plantio direto, o que também foi relatado por Suzuki et al. (2008) e Drescher et al. (2011).

Os valores baixos de RP, obtidos logo após a semeadura da soja (Figura 1), foram observados nas áreas escarificadas há $0,6,12,18$ e 24 meses. Os resultados também foram similares na avaliação realizada no final do ciclo de soja (Figura 2), na qual a escarificação manteve o perfil com menor RP até 18 meses (E18), a partir do qual foi constatada RP superior a 2,0 $\mathrm{MPa}$, valor frequentemente considerado crítico para o desenvolvimento radicular das culturas (Collares et al., 2006; Reichert et al., 2009). Embora a duração de alterações na Ds e na Pt tenha se mantido
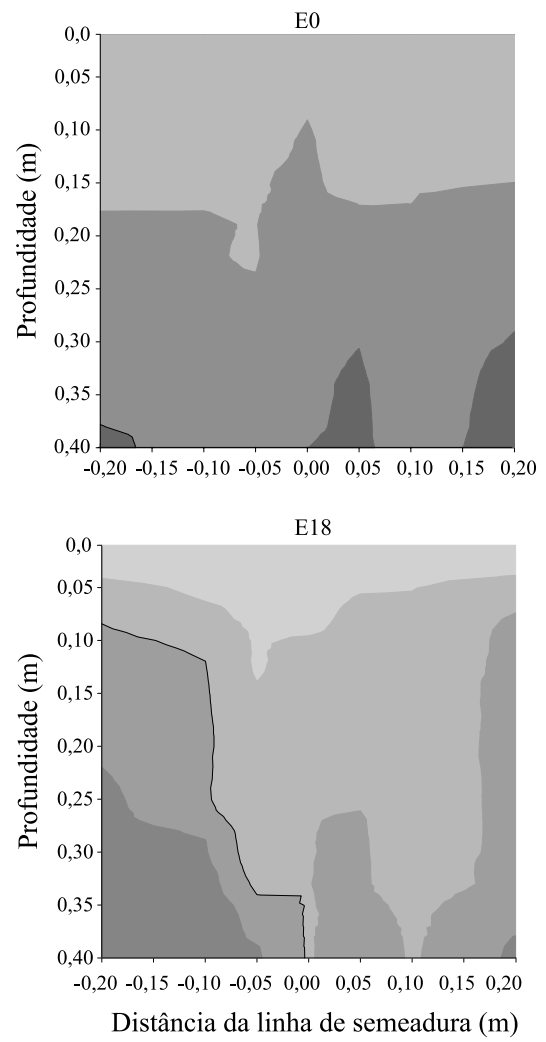
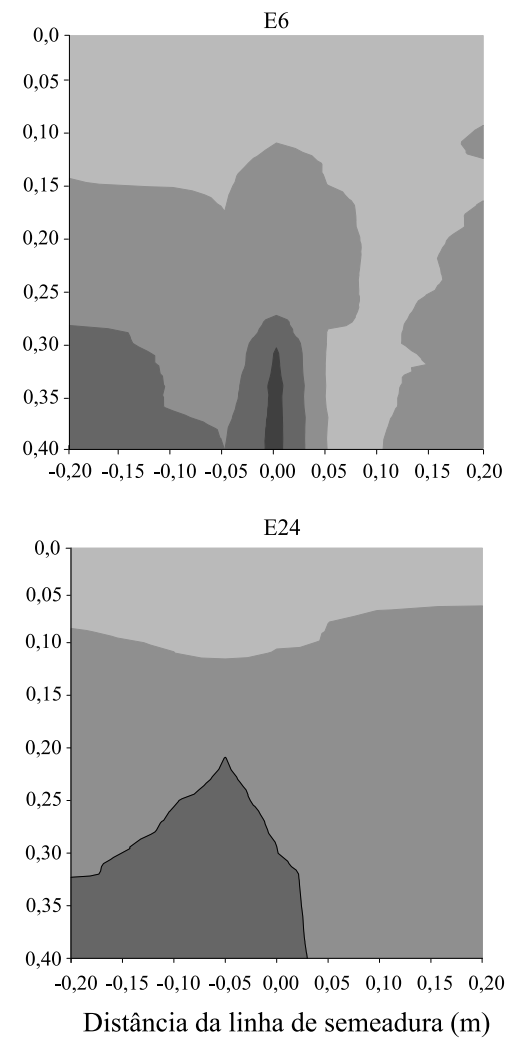
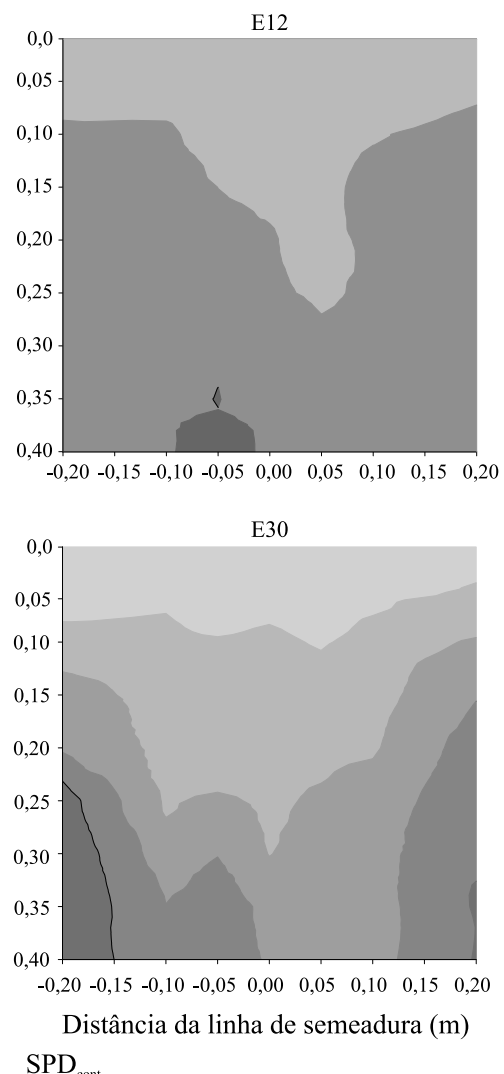
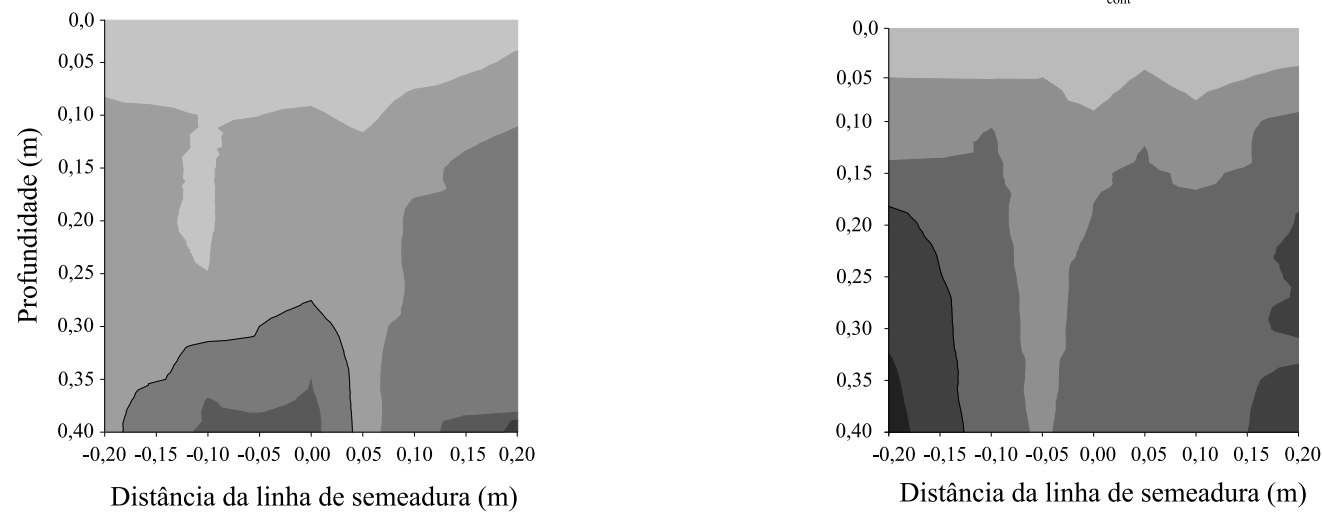

\begin{tabular}{|llll}
$\square$ & $\square \mathrm{MPa}$ \\
\hline & $\mathrm{MPa}$ & $\square$ & $\mathrm{MPa}$ \\
\hline
\end{tabular}

Figura 1. Resistência do solo à penetração medida aos 20 dias após a semeadura de soja (Glycine max), safra 2012/2013, em Latossolo Vermelho distrófico típico, manejado em sistema plantio direto contínuo há 27 anos $\left(\mathrm{SPD}_{\text {cont }}\right)$ e em $\mathrm{SPD}_{\mathrm{i}}$ escarificado mecanicamente há 0 (E0), 6 (E6), 12 (E12), 18 (E18), 24 (E24), 30 (E30) e 36 (E36) meses. 
por apenas uma safra agrícola, a duração de alterações na RP foi percebida por, no mínimo, 18 meses. Por isso, antes de realizar nova escarificação baseada apenas nos valores de Ds e Pt, é de suma importância verificar, também, os valores de RP, para avaliar se a RP indica compactação restritiva ao desenvolvimento radicular, uma vez que a resposta da planta dependerá da magnitude e da duração da resistência oferecida pelo solo (Bengough et al., 2006). Assim, a avaliação da RP serve como parâmetro melhor que a Ds e a Pt para apoiar a decisão de repetir ou não a escarificação mecânica do solo.

Para a condutividade hidráulica do solo saturado, o efeito da escarificação foi mantido até 18 meses (E18),
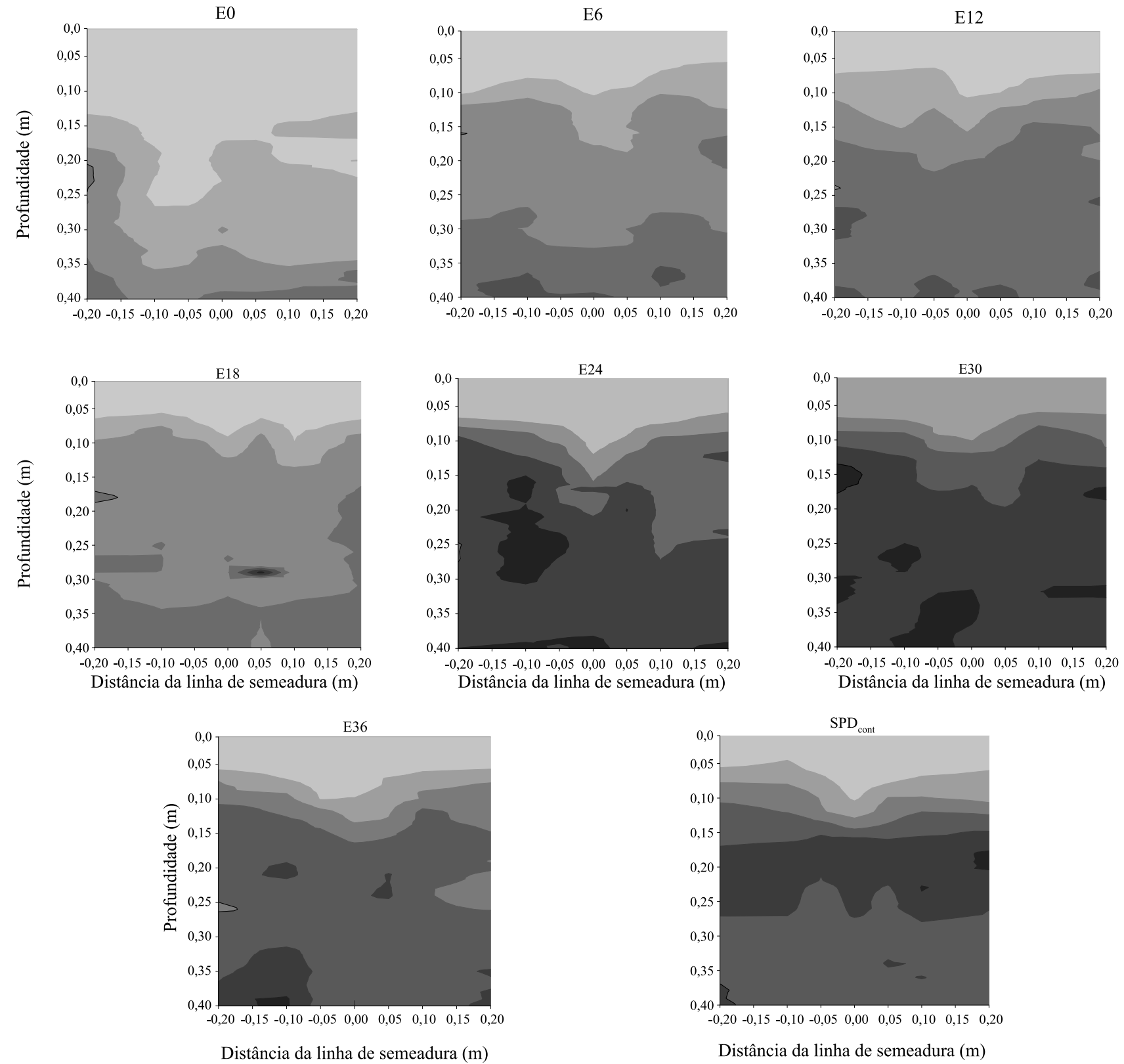

$0 \mathrm{MPa}$

$1 \mathrm{MPa}$

$3 \mathrm{MPa}$

Figura 2. Resistência do solo à penetração medida ao final do ciclo de soja (Glycine max), safra 2012/2013, em Latossolo Vermelho distrófico típico, manejado em sistema plantio direto contínuo há 27 anos $\left(\mathrm{SPD}_{\text {cont }}\right)$ e em SPD escarificado mecanicamente há 0 (E0), 6 (E6), 12 (E12), 18 (E18), 24 (E24), 30 (E30) e 36 (E36) meses. 

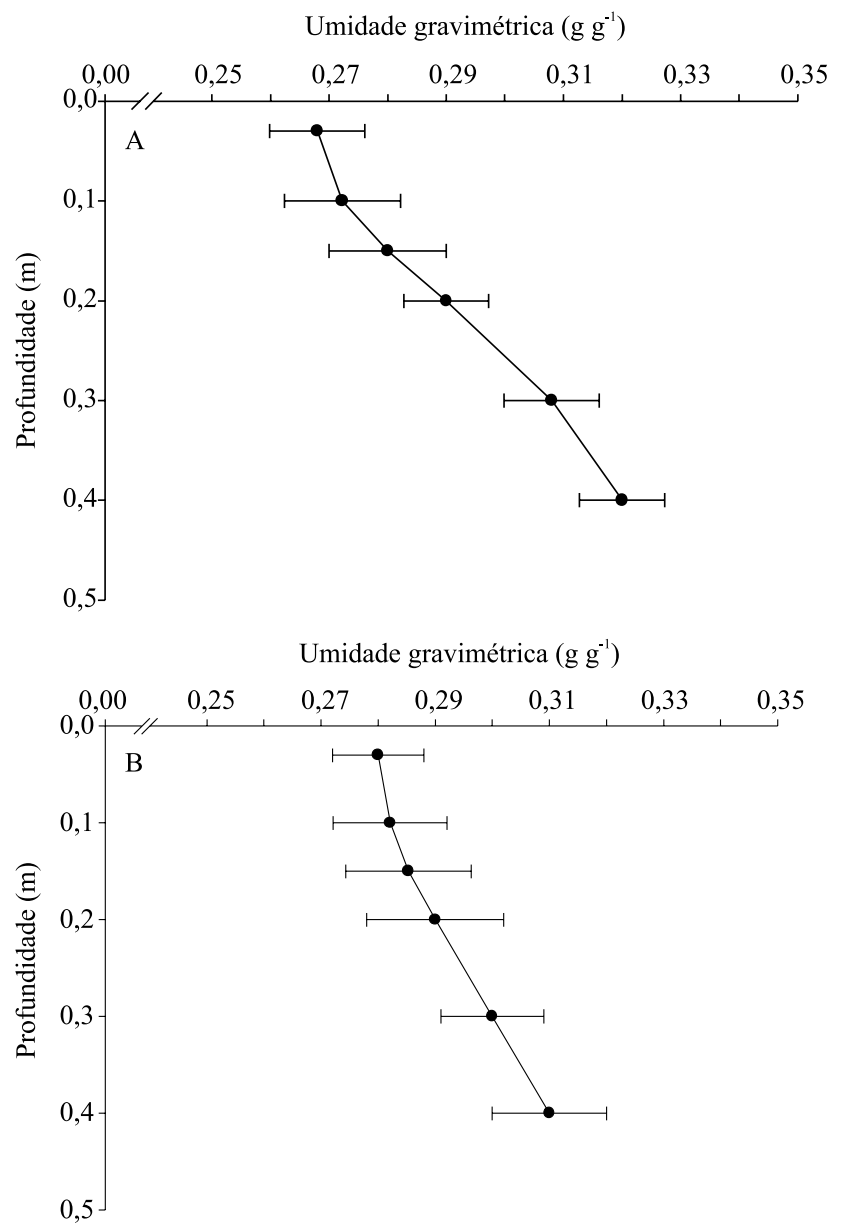

Figura 3. Conteúdo gravimétrico de água e desvio-padrão em Latossolo Vermelho distrófico típico, manejado em sistema plantio direto, no momento da avaliação da resistência do solo à penetração, no início, em 20/12/2012 (A), e no fim, em 18/4/2013 (B), do ciclo da cultura de soja (Glycine max). na camada de 0 a $0,07 \mathrm{~m}$, e até 24 meses (E24) na camada de 0,07 a $0,15 \mathrm{~m}$ de profundidade (Tabela 3 ). Isso indica que, para uma mesma propriedade do solo, a duração da escarificação também pode ser influenciada pela condição inicial de compactação. Dessa forma, tanto para Ks quanto para $\mathrm{Pt}$, microporosidade e Ds (Tabela 2), observou-se maior duração da escarificação na camada de 0,07 a $0,15 \mathrm{~m}$, na qual o grau de compactação foi superior a $90 \%$ em quase todos os tratamentos, exceto em E0. Esse comportamento também foi evidenciado por Drescher et al. (2011) e Nunes et al. (2014), que constataram manutenção do efeito da escarificação na densidade e na distribuição do tamanho de poros apenas na camada de 0,07 a 0,20 m, respectivamente, em Latossolo e Nitossolo, ambos de textura argilosa.

Assim como a Ks, a taxa de infiltração de água no solo também detectou as modificações na estrutura do solo promovidas pela escarificação. A taxa de infiltração (Figura 4) decresceu a um valor praticamente estável após 2 horas de infiltração ( $\mathrm{TIE}_{2 \text { horas }}$ ), o qual foi utilizado para a comparação dos tratamentos. Alterações na TIE $_{2 \text { horas }}$ foram percebidas até 24 meses após a escarificação (E24), apesar de terem sido mais expressivas nos primeiros 12 meses (E0 e E6). Portanto, não foram detectadas diferenças na $\mathrm{TIE}_{2 \mathrm{horas}}$ em períodos superiores há 24 meses (E30 e E36), em relação à testemunha ( $\left.\mathrm{SPD}_{\text {cont }}\right)$.

A duração dos efeitos da escarificação mecânica depende da propriedade do solo avaliada. As propriedades RP, Ks e $\mathrm{TIE}_{2 \mathrm{horas}}$ foram as mais sensíveis para detectar a duração das alterações na

Tabela 3. Condutividade hidráulica do solo saturado $\left(\mathrm{mm} \mathrm{h}^{-1}\right)$ em Latossolo Vermelho distrófico típico em diferentes camadas, manejado em sistema plantio direto contínuo há 27 anos $\left(\mathrm{SPD}_{\text {cont }}\right)$ e em SPD escarificado mecanicamente há $0(\mathrm{E} 0), 6(\mathrm{E} 6)$, 12 (E12), 18 (E18), 24 (E24), 30 (E30) e 36 (E36) meses ${ }^{(1)}$.

\begin{tabular}{|c|c|c|c|c|c|c|}
\hline \multirow[t]{2}{*}{ Tratamento } & \multicolumn{2}{|c|}{$0,0-0,07 \mathrm{~m}$} & \multicolumn{2}{|c|}{$0,07-0,15 \mathrm{~m}$} & \multicolumn{2}{|c|}{$0,15-0,25 \mathrm{~m}$} \\
\hline & Média & $\mathrm{SP}^{(2)}$ & Média & $\mathrm{SP}$ & Média & $\mathrm{SP}$ \\
\hline E0 & 780,5 & $117 \mathrm{a}$ & 281,7 & $99 a$ & 8,1 & $80^{\mathrm{ns}}$ \\
\hline E6 & 470,5 & $90 \mathrm{~b}$ & 182,3 & $97 \mathrm{a}$ & 9,7 & 54 \\
\hline E12 & 301,2 & $68 \mathrm{c}$ & 22,3 & $59 \mathrm{bc}$ & 7,9 & 67 \\
\hline E18 & 356,4 & $78 \mathrm{bc}$ & 25,7 & $50 \mathrm{c}$ & 40,7 & 89 \\
\hline E24 & 27,0 & $26 \mathrm{e}$ & 77,0 & $94 a$ & 4,9 & 43 \\
\hline E30 & 26,6 & $27 \mathrm{e}$ & 13,9 & $36 \mathrm{~d}$ & 6,5 & 53 \\
\hline E36 & 344,6 & $79 b c$ & 38,8 & $62 b$ & 10,7 & 63 \\
\hline$\underline{\mathrm{SPD}_{\text {cont }}}$ & 79,6 & $43 \mathrm{~d}$ & 11,2 & $31 \mathrm{~d}$ & 8,6 & 79 \\
\hline CV (\%) & 96,6 & - & 55,5 & - & 65,1 & - \\
\hline
\end{tabular}

${ }^{(1)}$ Médias seguidas de letras iguais, nas colunas, não diferem pelo teste t, a $5 \%$ de probabilidade. nenão significativo. ${ }^{(1)}$ SP, soma dos postos calculada no teste de Kruskal-Wallis. 
estrutura do solo provocadas pela escarificação. O uso da Ds e da Pt permite detectar alteração na ocupação do volume de solo por sólidos (Ds) ou vazios (Pt), para períodos de até uma safra agrícola após escarificação. Já o uso da Ks e da taxa de infiltração de água no solo permite detectar alteração no transporte de água no solo, para períodos mais prolongados.

Variáveis como Ks e taxa de infiltração estão diretamente relacionadas à geometria do espaço poroso do solo. De acordo com a Lei de Laplace, o raio dos poros altera a curvatura da superfície da
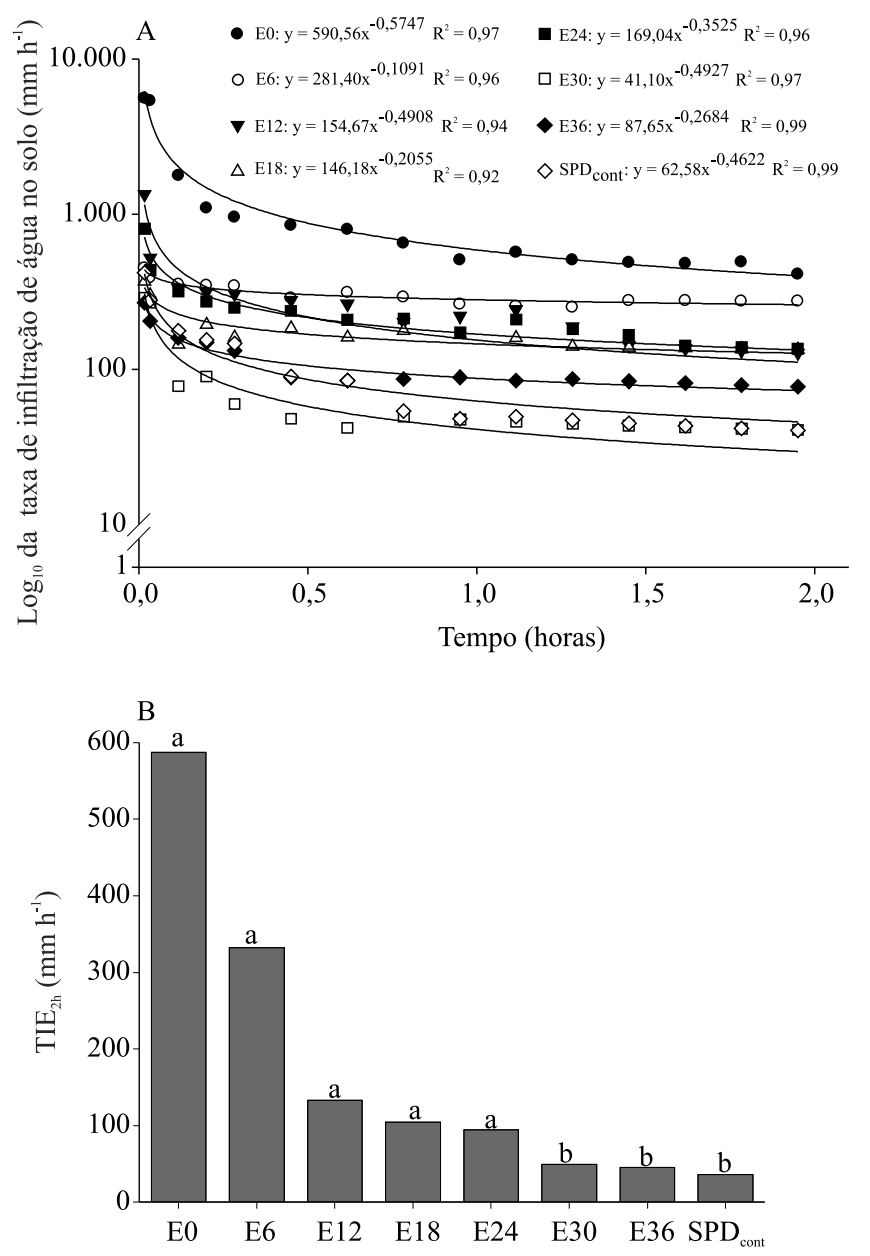

Figura 4. Taxa de infiltração de água em Latossolo Vermelho distrófico típico, manejado em sistema plantio direto contínuo há 27 anos $\left(\mathrm{SPD}_{\text {cont }}\right)$ e em SPD escarificado mecanicamente há 0 (E0), 6 (E6), 12 (E12), 18 (E18), 24 (E24), 30 (E30) e 36 (E36) meses, ao longo do tempo de medição (A) e ao final de 2 horas ( $\mathrm{TIE}_{2 \text { horas }}$ ) (B). Letras iguais, sobre as colunas, não diferem pelo teste de Dunnett, a $5 \%$ de probabilidade. água na interface ar-água, e a sucção em capilares é inversamente proporcional ao raio do poro, como estabelece a equação da capilaridade. Quanto ao fluxo de água, a Lei de Poiseuille determina que o volume de água que flui por unidade de tempo é proporcional à quarta potência do raio do tubo. Embora o sistema poroso do solo seja irregular e não possa ser descrito como um feixe de tubos paralelos, as equações da capilaridade e de Poiseuille são úteis para compreender a importância do raio aparente dos poros no transporte de água no solo. Desse modo, pequenas mudanças no raio dos poros por reconsolidação mudam pouco a Ds e a fração de classes de poros, mas podem mudar muito a taxa de infiltração (sucção capilar e fluxo saturado), a Ks e a TIE (fluxo saturado), o que confere maior sensibilidade a essas variáveis em resposta às alterações promovidas pelo manejo do solo.

Além disso, por haver maior ocupação do volume de solo por raízes após escarificação (Reichert et al., 2009; Nunes et al., 2015), mais bioporos podem permanecer no solo mesmo que haja reconsolidação de sua estrutura, pois a ausência de revolvimento favorece a estabilização de poros. Os bioporos facilitam os fluxos, tanto de ar quanto de água no solo, o que confere maiores taxas de infiltração de água. Portanto, para conhecer a duração da descompactação mecânica do solo, em condições de campo, a avaliação de propriedades relacionadas ao processo de transporte de água mostra-se mais adequada, uma vez que apresenta o efeito da escarificação mecânica por mais tempo.

\section{Conclusões}

1. A duração das alterações nas variáveis indicadoras do estado estrutural do solo, como densidade, porosidade total e macroporosidade do solo, após escarificação mecânica, é inferior a uma safra agrícola.

2. Propriedades do solo relacionadas ao transporte de água, como condutividade hidráulica e taxa de infiltração estável de água no solo, apresentam efeito da escarificação por até 24 meses e são as mais sensíveis para avaliar a duração da descompactação mecânica.

\section{Agradecimentos}

À Coordenação de Aperfeiçoamento de Pessoal de Nível Superior (Capes) e ao Conselho Nacional de Desenvolvimento Científico e Tecnológico (CNPq), pela concessão de bolsa e pelo apoio financeiro. 


\section{Referências}

ÁlVAREZ， C.R; DUGGAN, M.T.; CHAMORRO, E.R.; D'AMBROSIO, D.; TABOADA, M.A. Descompactación de suelos franco limosos en siembra directa: efectos sobre las propiedades edáficas y los cultivos. Ciencia del Suelo, v.27, p.159-169, 2009.

BENGOUGH, A.G.; BRANSBY, M.F.; HANS, J.; McKENNA, S.J.; ROBERTS, T.J.; VALENTINE, T.A. Root responses to soil physical conditions; growth dynamics from field to cell. Journal of Experimental Botany, v.57, p.437-447, 2006. DOI: 10.1093/ jxb/erj003.

BOTTA, G.F.; JORAJURIA, D.; BALBUENA, R.; RESSIA, M.; FERRERO, C.; ROSATTO, H.; TOURN, M. Deep tillage and traffic effects on subsoil compaction and sunflower (Helianthus annus L.) yields. Soil and Tillage Research, v.91, p.164-172, 2006. DOI: 10.1016/j.still.2005.12.011.

CANARACHE, A.; HORN, R.; COLIBAS, I. Compressibility of soils in a long term field experiment with intensive deep ripping in Romania. Soil and Tillage Research, v.56, p.185-196, 2000. DOI: 10.1016/S0167-1987(00)00143-4.

CHAMEN, W.C.T.; MOXEY, A.P.; TOWERS, W.; BALANA, B.; HALLETT, P.D. Mitigating arable soil compaction: a review and analysis of available cost and benefit data. Soil and Tillage Research, v.146, p.10-25, 2015. DOI: 10.1016/j.still.2014.09.011.

CLAESSEN, M.E.C. (Org.). Manual de métodos de análise de solo. 2.ed. rev. e atual. Rio de Janeiro: EMBRAPA-CNPS, 1997. $212 \mathrm{p}$.

COLLARES, G.L.; REINERT, D.J.; REICHERT, J.M.; KAISER, D.R. Qualidade física do solo na produtividade da cultura do feijoeiro num Argissolo. Pesquisa Agropecuária Brasileira, v.41, p.1663-1674, 2006. DOI: 10.1590/S0100-204X2006001100013.

COOPERATIVA DOS AGRICULTORES DE PLANTIO DIRETO. Plantio direto - reduzir custos de produção, melhorar a produtividade e preservar os recursos naturais. 2012. Disponível em: <http://www1.cooplantio.com.br/quem-somos/a-cooplantio. aspx>. Acesso em: 21 dez. 2012.

DENARDIN, J.E.; KOCHHANN, R.A.; FAGANELLO, A. 15 de abril dia nacional da conservação do solo: a agricultura desenvolvida no Brasil é conservacionista ou não? Boletim Informativo da Sociedade Brasileira de Ciência do Solo, v.36, p.10-15, 2011.

DRESCHER, M.S.; ELTZ, F.L.F.; DENARDIN, J.E.; FAGANELLO, A. Persistência do efeito de intervenções mecânicas para a descompactação de solos sob plantio direto. Revista Brasileira de Ciência do Solo, v.35, p.1713-1722, 2011. DOI: 10.1590/S0100-06832011000500026.

DRESCHER,M.S.;ELTZ,F.L.F.;DENARDIN,J.E.;FAGANELLO, A.; DRESCHER, G.L. Resistência à penetração e rendimento da soja após intervenção mecânica em Latossolo Vermelho sob plantio direto. Revista Brasileira de Ciência do Solo, v.36, p.1836-1844, 2012. DOI: 10.1590/S0100-06832012000600018.

FEDERAÇÃO BRASILEIRA DE PLANTIO DIRETO NA PALHA. Evolução da área cultivada no sistema de plantio direto na palha - Brasil. Disponível em: <http://www.febrapdp.
org.br/download/Ev_area_pd_brasil.pdf $>$. Acesso em: $10 \mathrm{dez}$. 2014.

GUBIANI, P.I.; GOULART, R.Z.; REICHERT, J.M.; REINERT, D.J. Crescimento e produção de milho associados com o intervalo hídrico ótimo. Revista Brasileira de Ciência do Solo, v.37, p.1502-1511, 2013. DOI: 10.1590/S0100-06832013000600007.

JIN, H.; HONGWEN, L.; XIAOYAN, W.; McHUGH, A.D.; WENYING, L.; HUANWEN, G.; KUHN, N.J. The adoption of annual subsoiling as conservation tillage in dryland maize and wheat cultivation in northern China. Soil and Tillage Research, v.94, p.493-502, 2007. DOI: 10.1016/j.still.2006.10.005.

KELLER, T.; LAMANDÉ, M.; PETH, S.; BERLI, M.; DELENNE, J.-Y.; BAUMGARTEN, W.; RABBEL, W.; RADJAÏ, F.; RAJCHENBACH, J.; SELVADURAI, A.P.S.; OR, D. An interdisciplinary approach towards improved understanding of soil deformation during compaction. Soil and Tillage Research, v.128, p.61-80, 2013. DOI: 10.1016/j.still.2012.10.004.

KLEIN, V.A.; BASEGGIO, M.; MADALOSSO, T. Indicadores da qualidade física de um Latossolo Vermelho distrófico típico sob plantio direto escarificado. Ciência Rural, v.39, p.2475-2481, 2009. DOI: 10.1590/S0103-84782009005000225.

MARCOLIN, C.D.; KLEIN, V.A. Determinação da densidade relativa do solo por uma função de pedotransferência para a densidade do solo máxima. Acta Scientiarum. Agronomy, v.33, p.349-354, 2011. DOI: 10.4025/actasciagron.v33i2.6120.

McDONALD, J.H. Handbook of biolological statistics. 3rd ed. Baltimore: Sparky House, 2014. 305p.

NUNES, M.R.; DENARDIN, J.E.; PAULETTO, E.A.; FAGANELLO, A.; PINTO, L.F.S. Effect of soil chiseling on soil structure and root growth for a clayey soil under no-tillage. Geoderma, v.259-260, p.149-155, 2015. DOI: 10.1016/j. geoderma.2015.06.003.

NUNES, M.R.; PAULETTO, E.A.; DENARDIN, J.E.; FAGANELLO, A.; PINTO, L.F.S.; SCHEUNEMANN, T. Persistência dos efeitos da escarificação sobre a compactação de Nitossolo sob plantio direto em região subtropical úmida. Pesquisa Agropecuária Brasileira, v.49, p.531-539, 2014. DOI: 10.1590/ S0100-204X2014000700005.

REICHERT, J.M.; KAISER, D.R.; REINERT, D.J.; RIQUELME, U.F.B. Variação temporal de propriedades físicas do solo e crescimento radicular de feijoeiro em quatro sistemas de manejo. Pesquisa Agropecuária Brasileira, v.44, p.310-319, 2009. DOI: 10.1590/S0100-204X2009000300013.

REINERT, D.J.; REICHERT, J.M. Coluna de areia para medir a retenção de água no solo - protótipos e teste. Ciência Rural, v.36, p.1931-1935, 2006. DOI: 10.1590/S0103-84782006000600044.

SANTOS, H.G. dos; JACOMINE, P.K.T.; ANJOS, L.H.C. dos; OLIVEIRA, V.A. de; LUMBRERAS, J.F.; COELHO, M.R.; ALMEIDA, J.A. de; CUNHA, T.J.F.; OLIVEIRA, J.B. de. Sistema brasileiro de classificação de solos. 3.ed. rev. e ampl. Brasília: Embrapa, 2013. 353p.

SCHJØNNING, P.; HECKRATH, G.; CHRISTENSEN, B.T. Threats to soil quality in Denmark: a review of existing knowledge in the context of the EU Soil Thematic Strategy. Tjele: 
Aarhus University, Faculty of Agricultural Sciences, 2009. 121p. (DJF Report Plant Science, 143).

SILVA, S.G.C.; SILVA, Á.P. da; GIAROLA, N.F.B.; TORMENA, C.A.; SÁ, J.C. de M. Temporary effect of chiseling on the compaction of a Rhodic Hapludox under no-tillage. Revista Brasileira de Ciência do Solo, v.36, p.547-555, 2012. DOI: 10.1590/S0100-06832012000200024.

SUZUKI, L.E.A.S.; REINERT, D.J.; REICHERT, J.M.; LIMA, C.L.R. de. Estimativa da susceptibilidade à compactação e do suporte de carga do solo com base em propriedades físicas de solos do Rio Grande do Sul. Revista Brasileira de Ciência do Solo, v.32, p.963-973, 2008. DOI: 10.1590/ S0100-06832008000300006.

TAVARES FILHO, J.; RALISCH, R.; GUIMARÃES, M.F.; MEDINA, C.C.; BALBINO, L.C.; NEVES, C.S.V.J. Método do perfil cultural para avaliação do estado físico de solos em condições tropicais. Revista Brasileira de Ciência do Solo, v.23, p.393-399, 1999. DOI: 10.1590/S0100-06831999000200022.

VIEIRA, M.L.; KLEIN, V.A. Propriedades físico-hídricas de um Latossolo Vermelho submetido a diferentes sistemas de manejo. Revista Brasileira de Ciência do Solo, v.31, p.1271-1280, 2007. DOI: $10.1590 /$ S0100-06832007000600006.

$\overline{\text { Recebido em } 12 \text { de outubro de } 2015 \text { e aprovado em } 14 \text { de janeiro de } 2016}$ 